\title{
Facteurs déterminant la productivité et la séquestration de carbone de Acacia auriculiformis A. Cunningham ex Benth. au Bénin
}

Jesugnon F. Murielle Féty TonouÉWA ${ }^{1}$ Éméline P. S. AssÉdÉ ${ }^{1,2}$

Samadori S. H. BıAOU ${ }^{1,2}$ Armand K. NATTA ${ }^{1,2}$

${ }^{1}$ Université de Parakou Laboratoire d’Écologie, de Botanique et de Biologie végétale 03 BP 125, Parakou Bénin

2 Université de Parakou Faculté d'Agronomie Département d'Aménagement et Gestion des Ressources Naturelles BP 123, Parakou

Bénin

Auteur correspondant / Corresponding author: Jesugnon F. Murielle Féty Tonouéwa tonouewam@gmail.com

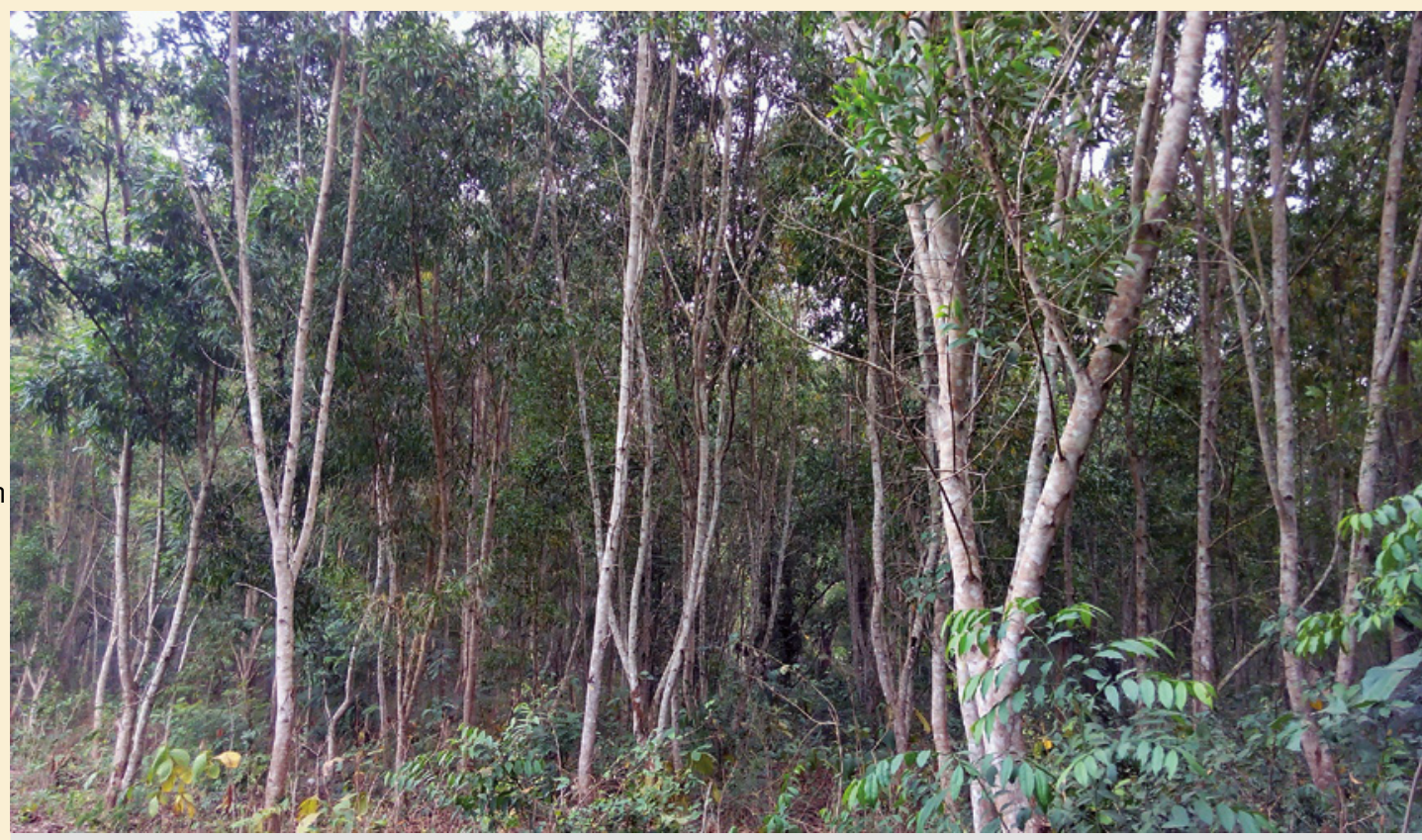

Photo 1.

Plantation à Acacia auriculiformis âgée de 5 ans à Ouedo. Photo J. F. M. F. Tonouéwa.

Doi : 10.19182/bft2019.342. a31787 - Droit d'auteur (C) 2019, Bois et Forêts des Tropiques (C Cirad - Date de soumission : 11 octobre 2018 ; date d'acceptation : 5 mars 2019 ; date de publication : 15 octobre 2019.

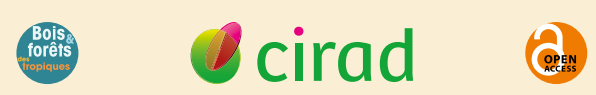

(c) $(1) \Theta$
Licence Creative Commons :

Attribution - Pas de Modification 4.0 International.

Attribution-NoDerivatives 4.0 International (CC BY-ND 4.0)
Citer l'article / To cite the article

Tonouéwa J. F. M. F., Assédé E. P. S., Biaou S. S. H., Natta A. K, 2019.

Facteurs déterminant la productivité et la séquestration de carbone de Acacia auriculiformis A. Cunningham ex Benth. au Bénin. Bois et Forêts des Tropiques, 342 : 17-28. Doi : https://doi.org/10.19182/bft2019.342. a31787 


\section{RÉSUMÉ}

\author{
Facteurs déterminant la productivité et \\ la séquestration de carbone de Acacia \\ auriculiformis A. Cunningham ex Benth. \\ au Bénin
}

Acacia auriculiformis est la troisième essence à croissance rapide la plus plantée au Bénin. Son bois, initialement destiné à l'énergie domestique, est de plus en plus utilisé comme bois d'œuvre. Ceci pourrait représenter une alternative durable à l'exploitation forestière des aires de conservation et contribuer à la séquestration de carbone. Cette étude avait pour objectif d'évaluer les facteurs qui influencent la production de bois et la séquestration de carbone des plantations de $A$. auriculiformis au Bénin. Douze plantations jeunes (4-7 ans), d'âge moyen (9-11 ans) et âgées (1529 ans), réparties sur sols ferrugineux, vertiques, ferrallitiques et sableux dans la zone climatique guinéenne, ont été étudiées. Les paramètres mesurés étaient l'accroissement moyen en diamètre, le diamètre à $1,30 \mathrm{~m}$, la hauteur du fût, la hauteur totale, la proportion de duramen et la proportion de bois sans défaut. La productivité est plus élevée sur sols ferrugineux et ferrallitiques, avec un accroissement moyen de $2,4 \mathrm{~cm}$ par an à 6 ans. Cet accroissement est significativement différent entre les arbres jeunes et les arbres âgés. La proportion de bois sans défaut est élevée sur sols ferrugineux. Par ailleurs, la séquestration de carbone est optimale à 20 ans. L'âge optimal pour la production de bois d'œuvre est d'environ 15 ans sur sols ferrugineux au Bénin. Le type de sol est le facteur le plus déterminant pour la production de bois de $A$. auriculiformis. Les sols ferrugineux représentent approximativement les deux tiers des sols rencontrés au Bénin, laissant présager de bonnes perspectives pour la filière de production de bois de $A$. auriculiformis. Toutefois, la caractérisation technologique de ce bois sur les différents sols au Bénin est nécessaire pour évaluer ses potentialités physico-mécaniques en bois de service.

Mots-clés : Acacia auriculiformis, accroissement, bois d'œuvre, carbone, type de sol, Afrique de l'Ouest.

\section{ABSTRACT}

\author{
Determining factors for Acacia \\ auriculiformis A. Cunningham ex Benth. \\ productivity and carbon sequestration \\ in Benin
}

Acacia auriculiformis is the third most frequently planted fast-growing tree species in Benin. Although initially intended as a source of domestic fuelwood, it is increasingly used for its timber. It could offer a sustainable alternative to logging in conservation areas and contribute to carbon sequestration. The aim of this study was to assess the factors that influence timber production and carbon sequestration in plantations of A. auriculiformis in Benin. The study was conducted in twelve plantations (young: 4-7 years, average-aged: 9-11 years, and mature: 15-29 years), distributed over ferruginous, vertisolic, ferralitic and sandy soils in the Guinean climatic zone. The parameters measured were average radial growth, diameter at $1.30 \mathrm{~m}$ height, bole height, total height, proportion of heartwood and proportion of blemish-free wood. Productivity was higher on ferruginous and ferralitic soils, with an average growth rate of $2.4 \mathrm{~cm} /$ year at 6 years of age. A significant difference in growth rates appeared between young and old trees. The proportion of blemish-free wood was highest on ferruginous soils. Optimum carbon sequestration was at 20 years of age. The optimum age for timber production in Benin was about 15 years for trees growing on ferruginous soils. Soil type is the most determining factor for the production of $A$. auriculiformis timber. Ferruginous soils account for about two thirds of all soil types found in Benin, which holds out good prospects for an $A$. auriculiformis timber sector. However, technical characterisation of A. auriculiformis wood grown on the different soil types in Benin is required to assess its physical and mechanical properties as a timber species.

Keywords: Acacia auriculiformis, growth, timber, carbon, soil type, West Africa.

\author{
Factores que determinan la \\ productividad y captura de carbono de \\ Acacia auriculiformis A. Cunningham \\ ex Benth. en Benín
}

Acacia auriculiformis es la tercera especie de crecimiento rápido más plantada en Benín. Su madera, inicialmente destinada a leña doméstica, cada vez se utiliza más como madera de construcción. Ello podría representar una alternativa sostenible a la explotación forestal de las zonas de conservación y contribuir a la captura de carbono. Este estudio tiene por objetivo evaluar los factores que influyen en la producción de madera y la captura de carbono de las plantaciones de $A$. auriculiformis en Benín. Se estudiaron doce plantaciones jóvenes (4-7 años), de mediana edad (9-11 años) y viejas (15-29 años), distribuidas en suelos ferruginosos, vérticos, ferralíticos y arenosos en la zona climática guineana. Los parámetros medidos eran el crecimiento medio en diámetro a 1,30 m, la altura del fuste, la altura total, la proporción de duramen y la proporción de madera sin defectos. La productividad es más elevada sobre suelos ferruginosos y ferralíticos, con un crecimiento medio de $2,4 \mathrm{~cm} / a$ ño en 6 años. Este crecimiento es significativamente diferente entre los árboles jóvenes y los árboles viejos. La proporción de madera sin defectos es elevada en suelos ferruginosos. Por otra parte, la captura de carbono es óptima a los 20 años. La edad óptima para la producción de madera de construcción es de unos 15 años en los suelos ferruginosos de Benín. El tipo de suelo es el factor más determinante para la producción de madera de $A$. auriculiformis. Los suelos ferruginosos representan aproximadamente dos tercios de los suelos encontrados en Benín, lo que permite presagiar buenas perspectivas para el sector de producción de madera de $A$. auriculiformis. Sin embargo, es necesario realizar la caracterización tecnológica de esta madera en los diferentes suelos de Benín para evaluar sus potencialidades fisicomecánicas como madera estructural.

Palabras clave: Acacia auriculiformis, crecimiento, madera de construcción, carbono, tipo de suelo, África occidental. 


\section{Introduction}

Les plantations de bois d'œuvre sont pertinentes dans les écosystèmes dégradés pour limiter les pressions sur les forêts naturelles (Sokpon et Ouinsavi, 2004 ; Owona Ndongo et al., 2009). Ces plantations sont également recherchées pour la séquestration du carbone et pour leur productivité en bois. Les essences forestières utilisées en plantation sont souvent sélectionnées sur la base de la qualité du bois (utilisation comme bois d'œuvre, bois de service ou bois énergie), de leurvitesse de croissance exprimée selon des paramètres de croissance tels l'accroissement moyen annuel en diamètre, le diamètre et la hauteur, et d'autres avantages qu'elles peuvent fournir (séquestration du carbone, résistance aux maladies et ravageurs, etc.).

Acacia auriculiformis est l'une des principales essences forestières de plantation. Son bois est utilisé dans la production de papier (Hai et al.., 2008), de bois d'œuvre (Hai et al., 2010 ; Griffin et al., 2011) et de bois énergie (Kataki et Konwer, 2002). Elle est utilisée en Asie, en Afrique et en Amérique (Gnahoua et Louppe, 2003). Son hybride obtenu avec Acacia mangium est également très utilisé pour la production de bois (Hung et al., 2016). En Afrique de l'Ouest, $A$. auriculiformis figure parmi les essences forestières d'intérêt majeur pour la fertilisation du sol et la production de bois énergie (N'Guessan et al., 2016).

Au Bénin, A. auriculiformis a été introduite en 1980 et est cultivée en peuplement pur ou en mélange avec d'autres essences forestières (Tandjiekpon et Dah-Dovonon, 1997 ; Fonton et al., 2002a). En peuplement pur, elle présenterait une meilleure productivité, ce qui justifie sa forte utilisation dans les forêts du Sud-Bénin (Fonton et al., 2002b). Toutefois, plusieurs facteurs peuvent influencer la croissance et la productivité des forêts. La croissance d'un arbre est fonction des contraintes aussi bien abiotiques que biotiques (Dobbertin, 2005). Le type de sol, les techniques de production, les émissions atmosphériques influencent significativement la production des plantations (Nemani et al., 2003 ; Duan et al., 2016). Seynave et al. (2008) ont établi la relation entre l'indice de fertilité et la productivité stationnelle du hêtre (Fagus sylvatica L.), et ont conclu que les paramètres du sol expliquent les variations de croissance des arbres de cette espèce. Selon Charru et al. (2010), la productivité d'un peuplement est fonction du stade de développement, de la densité et de la fertilité du site.

En plantation au Bénin, $A$. auriculiformis est prioritairement exploitée pour le bois énergie (Fonton et al., 2002a). Son emploi comme bois d'œuvre semble marginal et récent. Pourtant, vu l'importance de cette espèce dans les systèmes de production agricole et forestière au Bénin, son utilisation comme bois d'œuvre pourrait représenter une alternative intéressante pour freiner l'exploitation forestière dans les aires de conservation et forêts naturelles, et pour accroître le potentiel de séquestration de carbone des espaces boisés. Il est également important de diversifier les sources de bois dans le contexte actuel de changement climatique et de diminution des ressources forestières. Néanmoins, les principaux paramètres déterminant l'utilisation de $A$. auriculiformis comme bois d'œuvre (productivité, capacité de stockage de carbone, propriétés physiques et mécaniques) doivent être évalués.

Pour une utilisation en bois d'œuvre, les dimensions (diamètre, hauteur) d'un arbre représentent des paramètres de choix (Ahoba et al., 2016). Ainsi, les variations des classes de diamètre des arbres d'une parcelle peuvent permettre de prédire une utilisation donnée du bois. Pour une utilisation comme bois d'œuvre, un diamètre minimum d'exploitabilité est nécessaire (Sokpon et al., 2006). La connaissance des variations de classes de diamètre des arbres d'une parcelle permet donc d'envisager leur utilisation comme bois d'œuvre ou non. La proportion de duramen, la proportion de bois sans défauts sur un arbre sont autant de propriétés intéressantes pour le bois d'œuvre. L'utilisation d'un bois comme bois d'œuvre est également déterminée à partir de ses propriétés physiques et mécaniques (Gérard, 1991).

Cette étude se proposait de contribuer à la valorisation des ressources forestières au Bénin par l'analyse des facteurs influençant la productivité optimale en bois et le potentiel de séquestration de carbone des plantations de A. auriculiformis. De façon spécifique, il s'agissait :

- d'analyser la distribution par classe de diamètre des arbres en fonction de l'âge, du type de sol et de l'âge des éclaircies :

- de déterminer l'effet de l'âge et du type de sol sur l'accroissement moyen en diamètre, le pourcentage de duramen, la hauteur dominante et la proportion de bois sans défauts ;

- d'évaluer la dynamique du carbone stocké dans le bois en fonction de l'âge et du type de sol.

\section{Méthode}

\section{Présentation de l'espèce}

Acacia auriculiformis A. Cunningham ex Benth. est une essence forestière ligneuse à croissance rapide de la famille des Fabaceae et de la sous-famille des Mimosoideae (Maslin, 2014). Elle est originaire d'Australie, de Papouasie-Nouvelle-Guinée et d'Indonésie (Gnahoua et Louppe, 2003). Sur le plan commercial, elle est appelée northern black wattle, ou acacia. C'est un arbre à feuilles persistantes pouvant atteindre $30 \mathrm{~m}$ de hauteur et $60 \mathrm{~cm}$ de diamètre, distribué entre le niveau de la mer et $1000 \mathrm{~m}$ d'altitude, résistant à la sécheresse et s'adaptant à de nombreux sols (Gnahoua et Louppe, 2003).

Au Bénin, les plantations à $A$. auriculiformis étudiées sont sous la tutelle de la Direction Générale des Eaux, Forêts et Chasse (DGEFC). Les semences plantées sont issues de la provenance de Pahou au Bénin, elle-même établie à partir du site de Lilikopé au Togo. Les traitements sylvicoles sont similaires d'une zone à l'autre, et les plantations sont traitées en futaie car, en taillis, la régénération est faible (Tandjiekpon et Dah-Dovonon, 1997). 


\section{Milieu d'étude}

L'étude a été conduite au Sud-Bénin dans les localités de la Lama (latitude $6^{\circ} 51^{\prime}$ à $6^{\circ} 54^{\prime}$ Nord, longitude $2^{\circ} 05^{\prime}$ à $2^{\circ} 09^{\prime}$ Est), Ouedo (latitude 6 6 $30^{\prime}$ à 6 632' Nord, longitude $2^{\circ} 16^{\prime}$ à $2^{\circ} 14^{\prime}$ 'Est), Pahou (latitude 6 ${ }^{\circ} 22^{\prime}$ à $6^{\circ} 27^{\prime}$ 'Nord, longitude $2^{\circ} 8^{\prime}$ à $2^{\circ} 14^{\prime}$ Est) (département de l'Atlantique) et Sèmè-kpodji (latitude $6^{\circ} 22^{\prime}$ à $6^{\circ} 28^{\prime}$ Nord, longitude $2^{\circ} 28^{\prime}$ à $2^{\circ} 43^{\prime}$ Est) (département de l'Ouémé) (figure 1). Ces localités caractérisées par une mosaïque forêt-savane (Adomou, 2005), représentent les principaux sites de reboisement des plantations à $A$. auriculiformis de l'État. La végétation est dominée par des plantations, notamment de $A$. auriculiformis. Dans la Lama, toutefois, les plantations de Tectona grandis et Gmelina arborea sont les plus représentées.

Le climat des quatre localités est de type guinéen. Il est caractérisé par une grande saison pluvieuse de mars à juillet, une petite saison sèche en août, une petite saison pluvieuse de septembre à octobre et une grande saison sèche de novembre à février. La pluviométrie moyenne annuelle est de 1100 à $1150 \mathrm{~mm}$ et la température moyenne est de $27{ }^{\circ} \mathrm{C}$. Les plantations échantillonnées s'étendent sur des sols ferrugineux tropicaux à Pahou, sur des vertisols dans la Lama, sur des sols ferrallitiques à Ouedo et sur des sols sableux hydromorphes à Sèmè-kpodji (Volkoff et Willaime, 1976).
L'ensemble des plantations de $A$. auriculiformis étudiées sont sous forte pression anthropique, avec des coupes frauduleuses. À Sèmè-kpodji, il n'y a plus d'activités de production, du fait des prélèvements frauduleux des populations riveraines, principalement dans les plantations de 15 à 29 ans. À Ouedo, les activités de production sont intenses. Dans la Lama et à Pahou, il existe des plantations de différents âges.

\section{Collecte et analyse de données}

\section{Dispositif de collecte des données et échantillonnage}

La collecte des données a été réalisée sur la base d'échantillons portant sur plusieurs niveaux d'information (tableau I) :

- trois plantations d’âges divers par localité ;

- trois placeaux installés au hasard par plantation ;

- trois ou quatre arbres abattus par plantation (sauf à Ouedo), ayant un fût droit et présentant moins de déformations ou de blessures ;

- une rondelle de bois prélevée à $1,30 \mathrm{~m}$ pour chaque arbre abattu (sauf à Ouedo) ;

- quatre à sept barreaux de bois découpés par arbre abattu (sauf à Ouedo) ;

- une carotte de bois prélevée pour chacun des 21 arbres choisis au hasard dans chaque placeau.

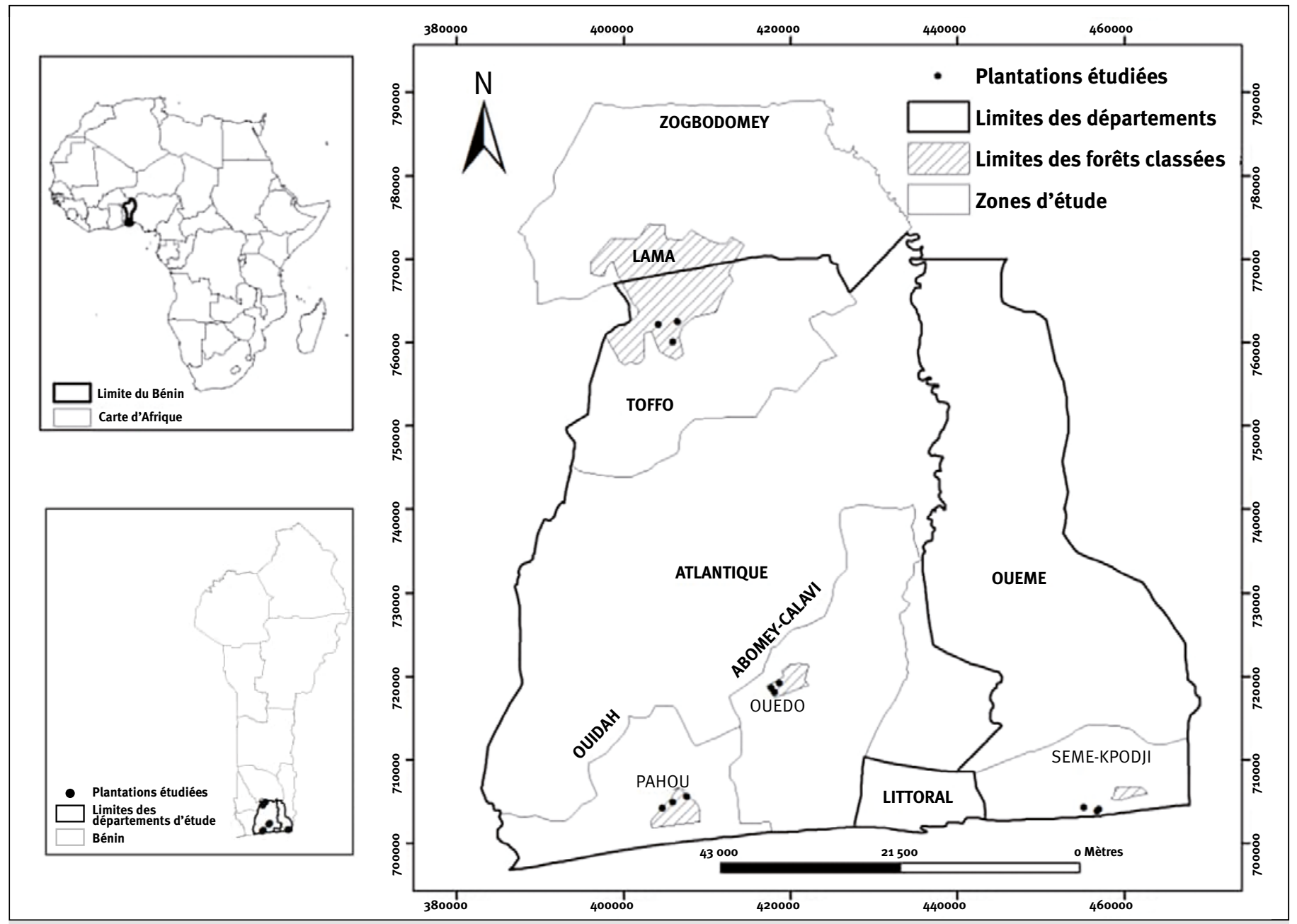

Figure 1.

Carte de la zone d'étude montrant les plantations étudiées dans le Sud-Bénin. 
Tableau I.

Caractéristiques des plantations et échantillons de bois prélevés.

\begin{tabular}{|c|c|c|c|c|c|c|c|c|c|c|c|c|}
\hline $\begin{array}{l}\text { Zone } \\
\text { Type de sol }\end{array}$ & & $\begin{array}{l}\text { Pahou } \\
\text { rugine }\end{array}$ & & & $\begin{array}{l}\text { Lama } \\
\text { ertiso }\end{array}$ & & Sable & $\begin{array}{l}\text { Sèmé } \\
\text { hydro }\end{array}$ & phes & & $\begin{array}{r}0 \\
\text { Ferrall }\end{array}$ & \\
\hline Âge (ans) & 6 & 9 & 15 & 7 & 9 & 11 & 27 & 27 & 29 & 4 & 5 & 6 \\
\hline Éclaircie (ans) & & & 9 & & & & 7 & 14 & & & & \\
\hline Placeaux (nombre) & 3 & 3 & 3 & 3 & 3 & 3 & 3 & 3 & 3 & 3 & 3 & 3 \\
\hline Arbres abattus (nombre) & 3 & 3 & 4 & 3 & 3 & 4 & 3 & 4 & 3 & 0 & 0 & 0 \\
\hline Rondelles à 1,30 m (nombre) & 3 & 3 & 4 & 3 & 3 & 4 & 3 & 4 & 3 & - & - & - \\
\hline Barreaux découpés (nombre) & 14 & 19 & 28 & 13 & 19 & 19 & 14 & 18 & 12 & - & - & - \\
\hline Carottes de bois (nombre) & 21 & 21 & 21 & 21 & 21 & 21 & 21 & 21 & 21 & 21 & 21 & 21 \\
\hline
\end{tabular}

En raison de la non-disponibilité des plantations de même âge au niveau des quatre zones, les plantations d’âges différents ont été échantillonnées. Les données collectées dans la plantation de Ouedo sont basées uniquement sur des méthodes non destructives.

\section{Classification des plantations et structure diamétrique de $\boldsymbol{A}$. auriculiformis}

Dans chacune des douze plantations (photo 1 ), trois placeaux circulaires de $1000 \mathrm{~m}^{2}$ chacun ont été installés. Les données recueillies étaient le diamètre à 1,30 m, la hauteur de fût et la hauteur totale des individus. Le diamètre et la hauteur des arbres ont été respectivement mesurés au moyen d'un ruban pi et d'un clinomètre Suunto.

Une typologie des plantations a été établie à partir d'une classification hiérarchique et d'un dendrogramme réalisés à l'aide du logiciel $R$ (version 3.5.0), sur la base des caractéristiques dendrométriques moyennes des peuplements : diamètre moyen des arbres à $1,30 \mathrm{~m}(\bar{d})$, hauteur fût moyenne, hauteur totale moyenne $(\bar{h})$ et densité des arbres $(\mathrm{N} / \mathrm{ha})$. Trois groupes homogènes de plantations ont été discriminés.

Les diamètres des arbres ont permis de déterminer les utilisations potentielles du bois d'A. auriculiformis et de le recommander comme bois d'œuvre ou bois énergie. De façon empirique, les exploitants se basent sur le diamètre des arbres de $A$. auriculiformis pour assurer cette affectation. La variation de la densité d'arbres par classe de diamètre à $1,30 \mathrm{~m}$ a été ajustée à la distribution de Weibull à trois paramètres. Le logiciel Minitab 14 a été utilisé pour calculer les paramètres de la distribution. La fonction $F(x)$ de densité de cette distribution (Rondeux, 1999) est :

$$
\mathrm{F}(\mathrm{x})=\frac{c}{b}\left(\frac{x-a}{b}\right)^{c-1} \exp ^{\left[-\left(\frac{x-a}{b}\right)^{c}\right]}
$$

où a est le paramètre de position, b le paramètre d'échelle ou de taille et c le paramètre de forme.

L'interprétation du type de la distribution a reposé sur la valeur du paramètre de forme (c) selon les modalités qui suivent (Husch et al., 2002) :

- c 1 : distribution en «J renversé ", caractéristique des peuplements multispécifiques ou inéquiennes ;

- $c=1$ : distribution exponentiellement décroissante, caractéristique des populations en extinction ;
- $1<c<3,6$ : distribution asymétrique positive ou asymétrique droite, caractéristique des peuplements monospécifiques avec prédominance d'individus jeunes ou de faible diamètre ;

- $c=3,6$ : distribution symétrique ; structure normale, caractéristique des peuplements à faible régénération ;

- c > 3,6 : distribution asymétrique négative ou asymétrique gauche, caractéristique des peuplements monospécifiques à prédominance d’individus âgés.

\section{Caractéristiques dendrométriques des plantations}

L'effet de l'âge et du type de sol a été testé sur l'accroissement moyen annuel en diamètre, le diamètre de l'arbre, la hauteur dominante, le pourcentage de duramen, la hauteur du fût, la hauteur totale et la proportion de bois sans défaut.

L'accroissement moyen annuel en diamètre $(a \bar{d})$ détermine la productivité des plantations (Dah-Dovonon et al., 2013). Il a été calculé comme suit :

$$
a \bar{d}=\frac{\pi}{4} \sum_{i=1}^{n} \frac{d i}{A}
$$

où $A$ est l'âge de l'arbre et $d i$ le diamètre de l'arbre $i$.

La hauteur dominante a été déterminée à partir de la moyenne des hauteurs des dix plus gros arbres au sein du placeau. Elle constitue un indice de fertilité stationnelle (N'Guessan et al., 2015).

Pour la détermination du pourcentage de duramen, deux types d'échantillons ont été prélevés : les rondelles (photo 2 b), récupérées sur 30 arbres abattus, et les carottes de bois (photo 2a), collectées sur 21 arbres sur pied sélectionnés dans chacun des 12 placeaux (tableau I).

Sur chaque arbre abattu, une rondelle de $10 \mathrm{~cm}$ d'épaisseur a été prélevée à 1,30 m. Sur chaque rondelle, huit rayons « moelle-limite écorce » et huit rayons « moelle-limite duramen » ont servi pour la mesure de la surface totale de la rondelle (S1) et la surface du duramen (S2). Le pourcentage de duramen ou bois de cœur (HW) a été ensuite déduit (Kokutse et al., 2004) selon les formules suivantes :

$$
\mathrm{S} 1=\frac{\pi \sum_{i=1}^{8} R i^{2}}{8} ; \quad \mathrm{S} 2=\frac{\pi \sum_{i=1}^{8} r^{2}}{8} ; \quad \mathrm{HW}=\frac{\mathrm{S} 2}{\mathrm{~S} 1} \times 100
$$
avec $R i=$ rayon moelle-limite écorce de l'arbre $i$ et $r i=$ rayon moelle-limite duramen de l'arbre $i$.

Pour les carottes de bois, prélevées à l'aide de la tarière 
de Pressler (photo 2a), le diamètre du duramen (Di) a été mesuré directement sur les carottes passant par la moelle de l'arbre. Le pourcentage duramen a été calculé à partir de la surface duramen (Rabhi et al., 2014) suivant la formule :

$$
\mathrm{HW}=\frac{\text { Shwi }}{\text { Gi }} \times 100
$$

où Shwi $=\prod \times\left(\frac{D i}{2}\right)^{2}, G i=\prod \times\left(\frac{d i}{2}\right)^{2}$, di étant le diamètre à $1,30 \mathrm{~m}$ de l'arbre $i$.

Un test de corrélation a été effectué entre le pourcentage de duramen obtenu sur les rondelles des arbres abattus et le pourcentage de duramen obtenu sur les carottes de bois des arbres dans l'ensemble des plantations.

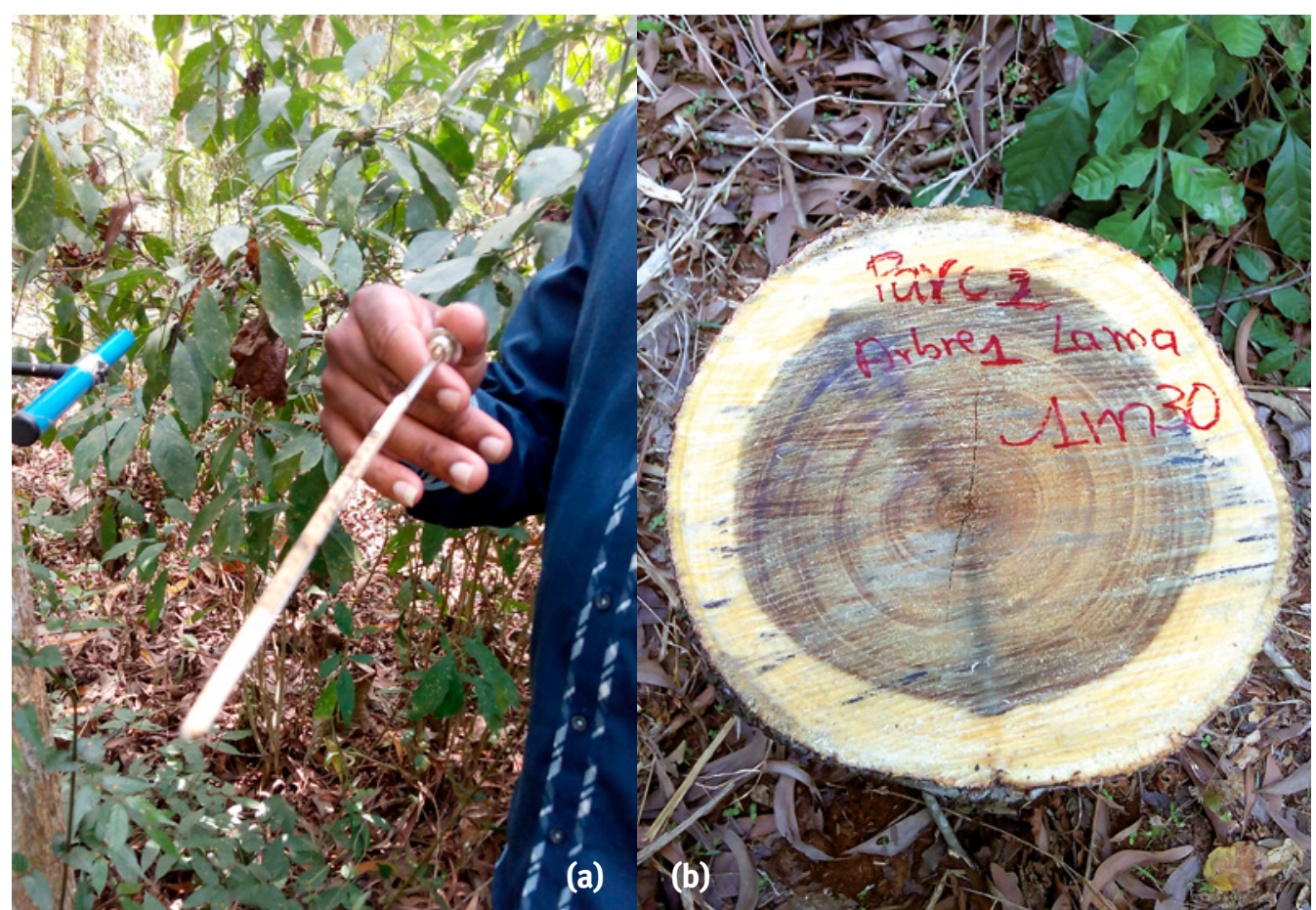

Photo 2.

Carotte (a) et rondelle de bois (b) de Acacia auriculifomis prélevées

dans la plantation de la Lama.

Photo J. F. M. F. Tonouéwa.

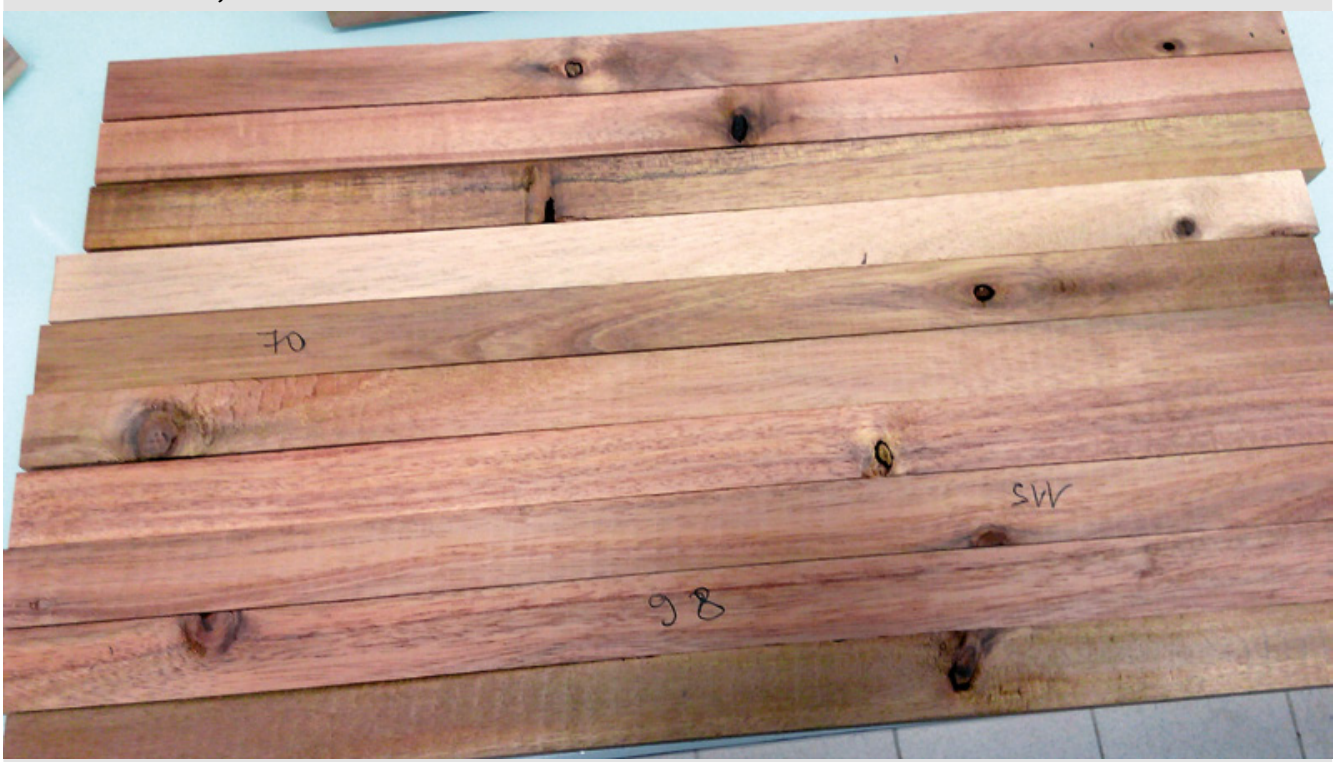

Photo 3.

Défauts à 1,30 m dénombrés sur les bois à Acacia auriculiformis.

Photo I. F. M. F. Tonouéwa.
Pour déterminer le pourcentage de bois sans défaut à $1,30 \mathrm{~m}$, une rondelle de $70 \mathrm{~cm}$ de hauteur a été découpée à $1,30 \mathrm{~m}$ sur chaque arbre abattu. Des barreaux $(4 \mathrm{~cm} \times 4 \mathrm{~cm} \times 60 \mathrm{~cm})$ ont été découpés dans la rondelle au niveau du duramen. Les barreaux présentant des défauts (nœuds, contrefils, pentes de fil ; photo 3) susceptibles d'influencer significativement la rupture en flexion ont été dénombrés.

Les statistiques descriptives présentant l'accroissement moyen en diamètre, le diamètre moyen à 1,30 m, le pourcentage de duramen, la hauteur totale, la densité d'arbres à l'hectare et la proportion de bois sans défauts à $1,30 \mathrm{~m}$ ont été présentées. Les accroissements moyens annuels en diamètre des plantations, le pourcentage de duramen, la hauteur dominante, la hauteur de fût, la hauteur totale ont été comparés en recourant au modèle à effet mixte comprenant toutes les variables explicatives et leurs interactions à l'aide du logiciel $\mathrm{R}$.

Dynamique du carbone stocké dans le bois en fonction de l'âge et du type de sol Le volume frais des échantillons de bois prélevés sur les barreaux à 1,30 m sur les arbres abattus a été calculé par poussée d'Archimède (le déplacement de volume d'eau dû à l'introduction de l'échantillon a été noté, les échantillons qui flottent ont été maintenus immergés). Ces échantillons ont ensuite été séchés à l'étuve à $103^{\circ} \mathrm{C}$ pendant trois jours. La masse sèche a été déterminée par pesée à l'aide d'une balance électronique. La masse sèche totale de l'arbre a été obtenue en la rapportant au volume total de l'arbre (Ostadhashemi et al., 2014). Le tarif de cubage décrit par Fonton et al. (2002a) sur des plantations à A. auriculiformis sur vertisols a été utilisé pour calculer le volume sur pied des arbres, suivant la formule :

$V=0,00259+0,407 d^{2} h$ avec $V=$ volume de l'arbre, $d=$ diamètre de l'arbre, $h=$ hauteur totale de l'arbre. 
À défaut d'un tarif de cubage pour chaque type de sol, nous avons utilisé le tarif établi pour $A$. auriculiformis sur vertisols au Bénin. Le taux de carbone est estimé entre 45 et $55 \%$ du taux de matière sèche avec une moyenne de $50 \%$ pour les espèces forestières (Peltier et al., 2007 ; Fonseca et al., 2011 ; Jha, 2018). Nous avons déduit le taux de carbone du bois de $A$. auriculiformis en considérant un taux moyen de carbone de $50 \%$. La relation entre l'âge des arbres et le taux de carbone stocké a été présentée graphiquement et ajustée à une fonction polynomiale.

\section{Résultats}

\section{Typologie et caractéristiques dendrométriques des plantations étudiées}

Les groupes de plantations discriminés ont été dénommés " types de plantation ». On distingue les plantations de 4 à 7 ans (plantations jeunes), de 9 à 11 ans (plantations d'âge moyen) et de 15 à 29 ans (plantations âgées) (figure 2).

La structure par classe de diamètre de l'ensemble des plantations s'ajuste bien à la distribution de Weibull avec un paramètre de forme compris entre 1 et 3,6. Elle correspond à une distribution asymétrique positive, ce qui traduit que les plantations sont à prédominance d'individus jeunes ou de faible diamètre (figures 3, 4 et 5).

Les plantations jeunes présentent un effectif élevé d'arbres entre 10 et $15 \mathrm{~cm}$ de diamètre (figure 3), quel que soit le type de sol. Les plantations jeunes sur sols ferrugineux et ferrallitiques présentent une distribution semblable. Celles établies sur vertisols présentent moins d'individus de gros diamètre que les plantations sur sols ferrugineux et ferrallitiques.

$\mathrm{Au}$ sein des plantations d'âge moyen, les arbres de 15 à $20 \mathrm{~cm}$ de diamètre sont davantage représentés, quel que soit le type de sol (figure 4). Toutefois, sur sols ferrugineux, des arbres de diamètre compris entre 30 et $35 \mathrm{~cm}$ sont présents, alors qu'ils restent absents dans les plantations de même âge sur vertisols.

Les plantations âgées présentent une distribution par classe de diamètre similaire, quel que soit le type de sol, mais les plantations ayant subi une éclaircie disposent d'une densité élevée d'arbres de diamètre compris entre 25 et $30 \mathrm{~cm}$, et celles n'ayant subi aucune éclaircie ont une densité élevée d'arbres de diamètre

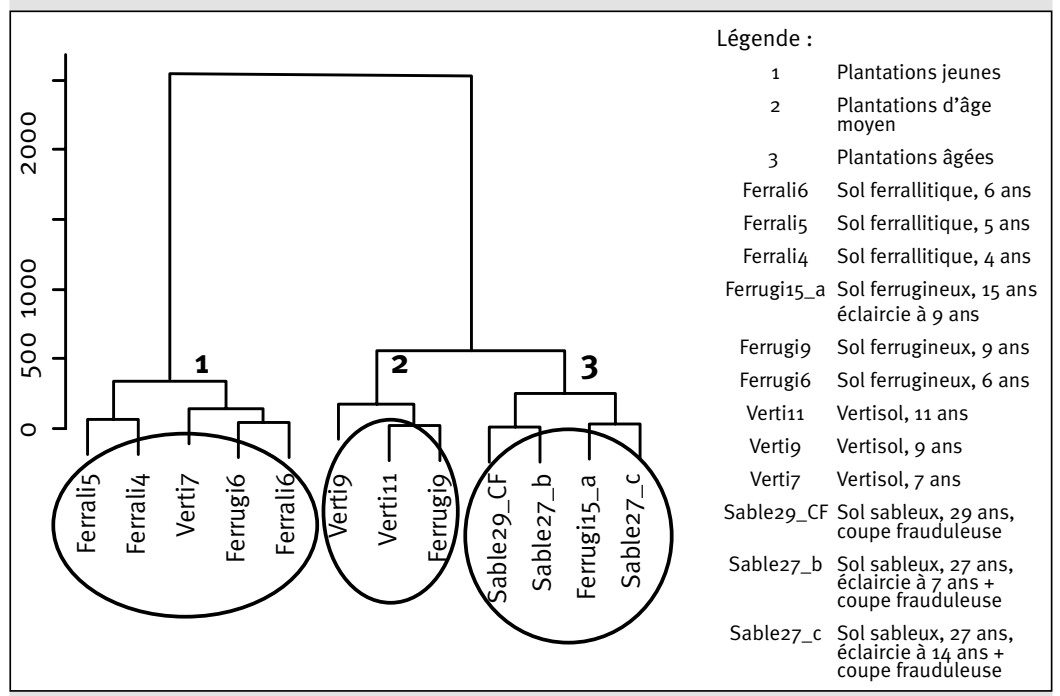

Figure 2.

Dendrogramme résultant de la classification des plantations étudiées.
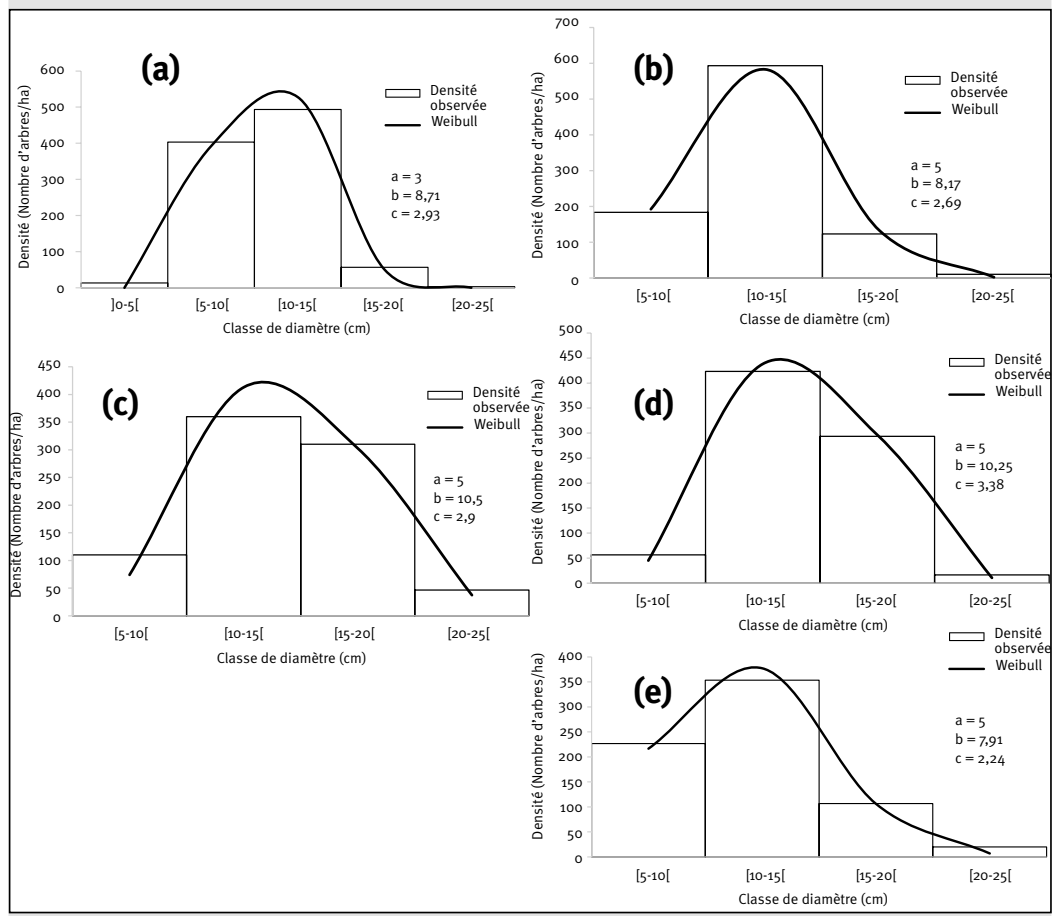

Figure 3.

Distribution par classe de diamètre $(\mathrm{cm})$ des plantations jeunes étudiées, ajustée à la distribution de Weibull. a : sols ferrallitiques 4 ans ; b : sols ferrallitiques 5 ans ; $c$ : sols ferrallitiques 6 ans; $d$ : sols ferrugineux 6 ans; e : vertisols 7 ans.

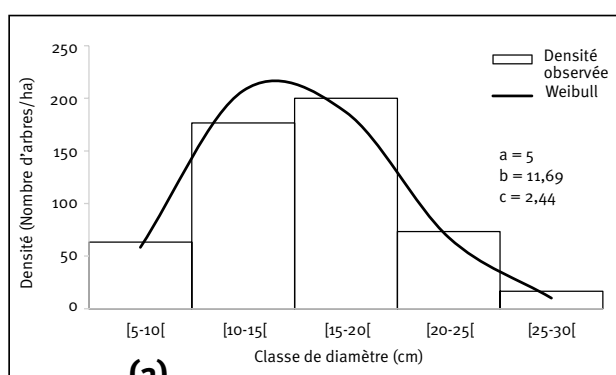

(a)

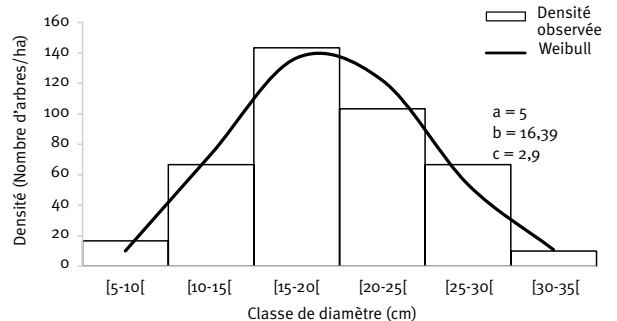

(b)

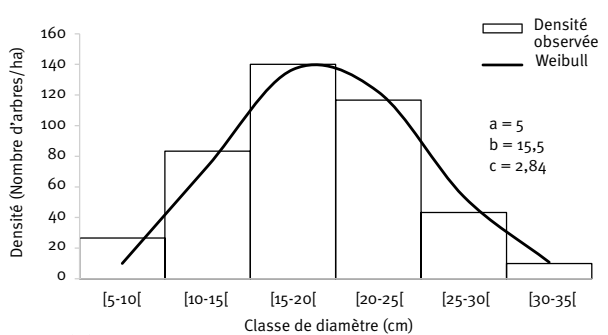

(c)

Figure 4.

Distribution par classe de diamètre $(\mathrm{cm})$ des plantations d'âge moyen étudiées, ajustée à la distribution de Weibull. $\mathrm{a}$ : vertisols 9 ans; $\mathrm{b}$ : sols ferrugineux 9 ans ; $\mathrm{c}$ : vertisols 11 ans. 

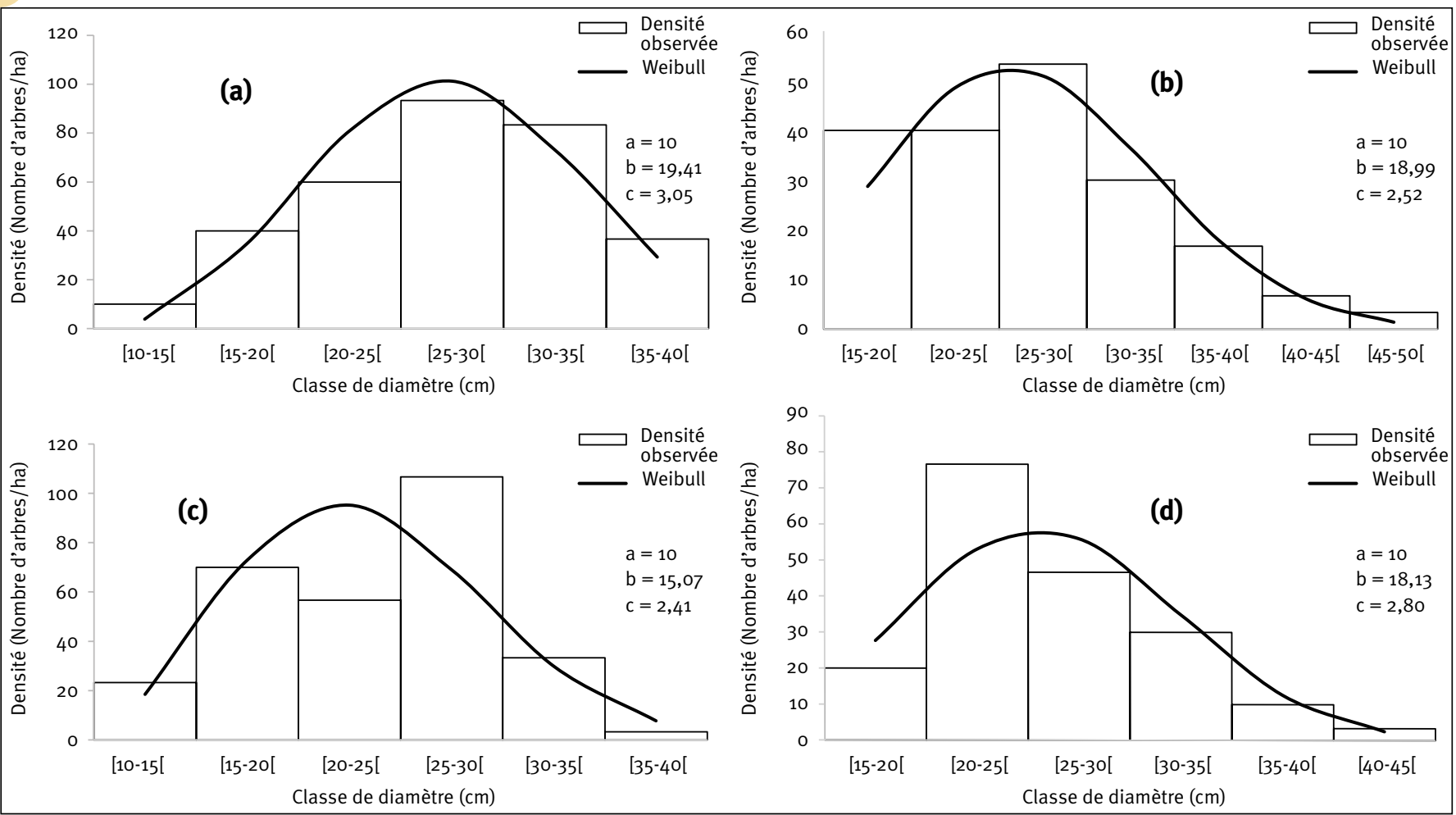

Figure 5.

Distribution par classe de diamètre $(\mathrm{cm})$ des plantations âgées étudiées, ajustée à la distribution de Weibull : a : sols ferrugineux, 15 ans avec éclaircie à 9 ans ; b : sols sableux, 27 ans avec éclaircie à 7 ans ; c : sols sableux, 27 ans avec éclaircie à 14 ans ; $d$ : sols sableux, 29 ans sans éclaircie.

situé entre 20 et $25 \mathrm{~cm}$ (figure 5). Toutefois, une classe supplémentaire de diamètres allant de 45 à $50 \mathrm{~cm}$ se manifeste, sur sols sableux, au sein des plantations âgées ayant subi une éclaircie.

Les arbres sur sols ferrugineux et ferrallitiques présentent un diamètre à $1,30 \mathrm{~m}$ et une hauteur supérieurs à ceux sur les autres types de sols (tableau II). Les arbres sur sols ferrugineux et ferrallitiques présentent un accroissement en diamètre plus élevé que ceux sur vertisols et sols sableux. Toutefois, l'accroissement annuel diminue quand l'âge augmente (tableau II et III).

Le type de sol et l'âge déterminent significativement l'accroissement des plantations. II n'y a pas de différence significative entre l'accroissement en diamètre des arbres de plantations d'âge moyen et ceux des plantations âgées. En revanche, une différence significative apparaît entre l'accroissement des plantations âgées et celles jeunes $\left(\mathrm{t}\right.$-value $=-8,365 ; \mathrm{p}$-value $\left.=0,000^{* \star *}\right)$.

L'indice de fertilité, dont dépend la hauteur, est le plus élevé sur sols ferrugineux (Pahou), puis sur sols ferrallitiques (Ouedo) et vertisols (Lama), et est moindre sur sols sableux (Sèmè-kpodji). Les hauteurs des arbres varient significativement d'un type de sol à l'autre, et d'une classe d'âge à l'autre (tableau III).

Les plantations âgées n'ayant subi aucune éclaircie présentent un pourcentage de bois sans défauts plus faible $(33,3 \%)$ que les autres types de plantations.
Le duramen est déjà présent sur les arbres de quatre ans (tableau II). Le type de sol influence significativement le pourcentage de duramen sur les carottes de bois. Le pourcentage de duramen des carottes est semblable sur vertisols et sols sableux (tableau III). Ce pourcentage sur vertisols est significativement différent de ceux sur sols ferrugineux et ferrallitiques. Le pourcentage de duramen est faible sur sols ferrugineux pour les plantations jeunes, et élevé sur vertisols. La corrélation entre le pourcentage de duramen sur les carottes et sur les rondelles est positive et très significative $\left(r=0,33 ; p\right.$-value $\left.=0,000^{\star \star \star}\right)$.

\section{Séquestration de carbone dans le bois de $A$. auriculiformis}

La quantité de carbone stockée dans l'arbre varie avec le type de sol et l'âge des plantations (figure 6). La quantité de carbone stockée sur sols ferrugineux est supérieure à celle sur vertisols. À neuf ans, un stockage de carbone de $0,08 \mathrm{t} / \mathrm{arbre} / \mathrm{ha}$ apparaît sur sols ferrugineux, contre $0,05 \mathrm{t} / \mathrm{arbre} / \mathrm{ha}$ sur vertisols. La non-disponibilité de plantations jeunes sur sols sableux limite la comparaison du carbone stocké sur ce type de sol avec ceux sur vertisols et sols ferrugineux. Entre 27 et 29 ans, le taux de carbone varie entre 0,13 et $0,18 \mathrm{t} / \mathrm{arbre} / \mathrm{ha}$ sur sols sableux. Globalement, la quantité de carbone stockée par $A$. auriculiformis augmente de 6 à 15 ans et diminue entre 22 et 


\section{Tableau II.}

Caractéristiques dendrométriques des plantations à Acacia auriculiformis étudiées.

\begin{tabular}{|c|c|c|c|c|c|c|c|c|c|c|c|c|c|c|c|c|c|c|}
\hline \multirow[t]{2}{*}{ Type de sol } & \multirow[t]{2}{*}{ Âge } & \multirow[t]{3}{*}{. 든 } & \multicolumn{2}{|c|}{$\begin{array}{l}\text { Accroissement } \\
\text { moyen annuel } \\
\text { en diamètre } \\
(\mathrm{cm} / \mathrm{an})\end{array}$} & \multicolumn{2}{|c|}{$\begin{array}{l}\text { Diamètre } \\
\text { à } 1,30 \mathrm{~m} \\
(\mathrm{~cm})\end{array}$} & \multicolumn{2}{|c|}{$\begin{array}{c}\text { Pourcentage } \\
\text { duramen } \\
\text { carotte (\%) }\end{array}$} & \multicolumn{2}{|c|}{$\begin{array}{l}\text { Pourcentage } \\
\text { duramen } \\
\text { rondelle (\%) }\end{array}$} & \multicolumn{2}{|c|}{$\begin{array}{l}\text { Hauteur } \\
\text { dominante } \\
\text { (m) }\end{array}$} & \multicolumn{2}{|c|}{$\begin{array}{l}\text { Hauteur } \\
\text { totale }(\mathrm{m})\end{array}$} & \multicolumn{2}{|c|}{$\begin{array}{l}\text { Hauteur } \\
\text { de fût }(m)\end{array}$} & \multirow[t]{2}{*}{$\begin{array}{c}\% \text { BD } \\
\text { à } \\
1,30 \\
m\end{array}$} & \multirow[t]{2}{*}{ N/ha } \\
\hline & & & m & se & m & se & $\mathrm{m}$ & se & $\mathrm{m}$ & se & m & se & m & se & m & se & & \\
\hline Ferrugineux & 6 & & 2,37 & 0,03 & 14,23 & 0,2 & 36,03 & 1,84 & 39,58 & 5,53 & 16,23 & 0,25 & 14,18 & 0,11 & 8,54 & 0,1 & 57,14 & 790 \\
\hline Vertisol & 7 & & 1,71 & 0,03 & 11,98 & 0,23 & 45,42 & 2,47 & 53,23 & 5,16 & 14,95 & 0,27 & 13,58 & 0,1 & 8,42 & 0,1 & 46,15 & 707 \\
\hline Ferrallitique & 6 & & 2,4 & 0,04 & 14,39 & 0,22 & 44,53 & 2,82 & - & - & 15,21 & 0,22 & 13,17 & 0,11 & 9,61 & 0,1 & - & 827 \\
\hline Ferrallitique & 5 & & 2,46 & 0,03 & 12,29 & 0,08 & 37,8 & 2,48 & - & - & 13,93 & 0,22 & 12,08 & 0,09 & 8,88 & 0,08 & - & 910 \\
\hline Ferrallitique & 4 & & 2,69 & 0,04 & 10,78 & 0,17 & 34,46 & 2,38 & - & - & 13,05 & 0,19 & 11,02 & 0,08 & 7,8 & 0,08 & - & 970 \\
\hline Ferrugineux & 9 & & 2,18 & 0,06 & 19,59 & 0,51 & 36,26 & 3,7 & 52,1 & 2,64 & 18,08 & 0,29 & 16,04 & 0,23 & 10,16 & 0,19 & 52,63 & 407 \\
\hline Vertisol & 9 & & 1,7 & 0,04 & 15,36 & 0,36 & 48,87 & 1,85 & 51,43 & 2,66 & 17,7 & 0,2 & 15,53 & 0,11 & 11,77 & 0,15 & 63,16 & 530 \\
\hline Vertisol & 11 & & 1,71 & 0,04 & 18,8 & 0,48 & 49,17 & 2,43 & 58,1 & 3,03 & 18,68 & 0,12 & 17,35 & 0,14 & 9,84 & 0,11 & 63,16 & 420 \\
\hline Ferrugineux & 15 & $\mathrm{a}$ & 1,82 & 0,04 & 27,34 & 0,65 & 43,7 & 3,89 & 61,26 & 6,96 & 21,96 & 0,19 & 19,21 & 0,29 & 12,46 & 0,24 & 60,71 & 323 \\
\hline Sableux & 29 & CF & 0,91 & 0,03 & 26,34 & 0,89 & 59,7 & 0,43 & 56,97 & 3,09 & 18,27 & 0,16 & 17,54 & 0,15 & 11,69 & 0,18 & 33,33 & 187 \\
\hline Sableux & 27 & $\mathrm{~b}$ & 0,99 & 0,04 & 26,81 & 0,95 & 50,53 & 3,36 & 56,08 & 2,96 & 16,47 & 0,27 & 16,1 & 0,18 & 10,14 & 0,15 & 50 & 190 \\
\hline $\begin{array}{l}\text { CF : co } \\
C: \text { éclé } \\
\text { sans d }\end{array}$ & 27 & c & 0,86 & : écl & 23,34 & 0,64 & 50,36 & 3,43 & 52,99 & 3,84 & 16,81 & 0,14 & 15,78 & 0,14 & 10,25 & 0,14 & 50 & 293 \\
\hline
\end{tabular}

29 ans. La relation entre la quantité de carbone stockée (y) dans l'arbre suivant l'âge $(x)$ des plantations correspond à une fonction polynomiale du second degré de la forme : $y=-0,0008 x^{2}+0,0351 x-0,1741\left(R^{2}=0,861\right)$. L'âge seuil de carbone maximal stockable est environ de 20 ans sur l'ensemble des sols étudiés et le carbone seuil est en moyenne de 0,2 t/arbre/ ha.

\section{Discussion}

Les arbres se développant sur sols ferrugineux présentent davantage d'individus de gros diamètre que ceux sur vertisols. Le type de sol et la pratique des éclaircies semblent donc déterminer la structure diamétrique des plantations. Les sols ferrugineux représentant les deux tiers des sols rencontrés au Bénin (FAO, 2002), la production du bois de $A$. auriculiformis au Bénin apparaît comme une filière prometteuse. Toutefois, la détermination des caractéristiques dendrométriques de cette essence dans les autres zones climatiques du Bénin comportant des sols ferrugineux permettra de préciser l'effet concomitant du climat sur la productivité de $A$. auriculiformis.

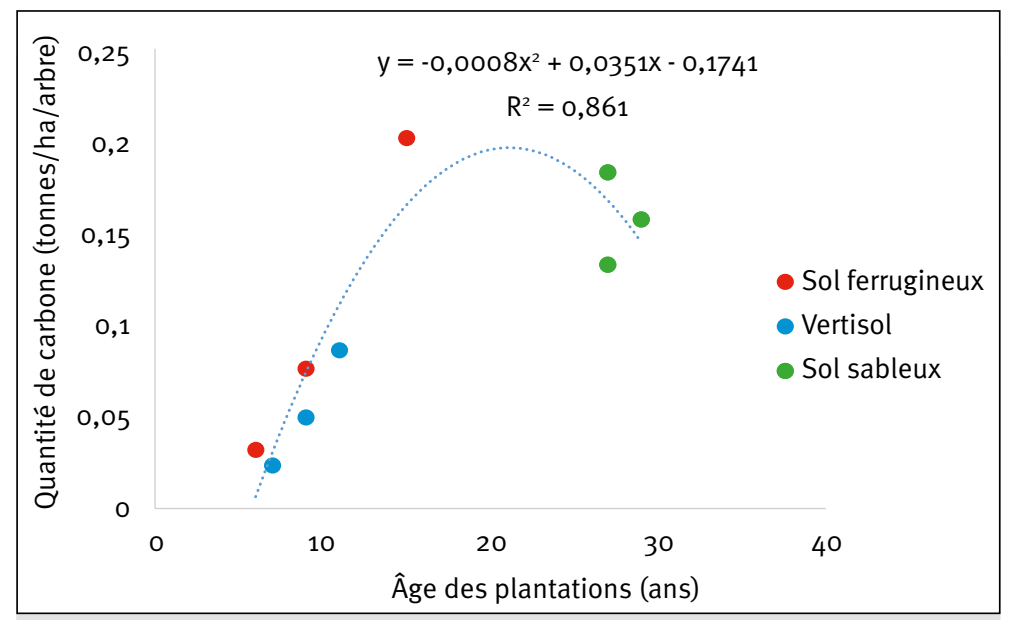

Figure 6.

Variation de la quantité de carbone stockée annuellement par hectare en fonction du type de sol et de l'âge des plantations. 


\section{Tableau III.}

Résultats du modèle mixte d'analyse de l'accroissement moyen en diamètre, du pourcentage duramen carotte, de la hauteur dominante, de la hauteur totale et de la hauteur fût en fonction du type de sol et du type de plantation.

\begin{tabular}{|c|c|c|c|c|c|c|c|c|c|c|c|c|c|c|c|c|c|c|c|c|}
\hline \multirow[b]{2}{*}{ Intercept } & \multicolumn{3}{|c|}{$\begin{array}{l}\text { Accroissement moyen } \\
\text { annuel en diamètre }\end{array}$} & \multirow[b]{2}{*}{$\begin{array}{l}\text { p-value } \\
0,000^{\star \star \star}\end{array}$} & \multicolumn{3}{|c|}{$\begin{array}{l}\text { Pourcentage duramen } \\
\text { carotte }\end{array}$} & \multirow{2}{*}{ p-value } & \multicolumn{3}{|c|}{ Hauteur dominante } & \multirow[b]{2}{*}{$p$-value } & \multicolumn{3}{|c|}{ Hauteur totale } & \multirow[b]{2}{*}{$\mathrm{p}$-value } & \multicolumn{2}{|c|}{ Hauteur fût } & \multirow[b]{2}{*}{ t-value } & \multirow[b]{2}{*}{$p$-value } \\
\hline & $\begin{array}{l}\text { Estim } \\
1,71\end{array}$ & $\begin{array}{c}\text { se } \\
0,041\end{array}$ & $\begin{array}{l}\text { t-value } \\
41,52\end{array}$ & & $\begin{array}{l}\text { Estim } \\
45,43\end{array}$ & $\begin{array}{c}\text { se } \\
2,24\end{array}$ & $\begin{array}{l}\text { t-value } \\
20,26\end{array}$ & & $\begin{array}{l}\text { Estim } \\
14,95\end{array}$ & $\begin{array}{c}\text { se } \\
0,24\end{array}$ & $\begin{array}{l}\text { t-value } \\
61,34\end{array}$ & & $\begin{array}{l}\text { Estim } \\
13,57\end{array}$ & $\begin{array}{c}\text { se } \\
0,13\end{array}$ & $\begin{array}{l}\text { t-value } \\
105,4\end{array}$ & & $\begin{array}{l}\text { Estim } \\
8,42\end{array}$ & $\begin{array}{c}\text { se } \\
0,11\end{array}$ & & \\
\hline \multicolumn{21}{|c|}{ Effet du type de plantation } \\
\hline $\begin{array}{l}\text { Plantations } \\
\text { jeunes } \\
\text { (catégorie de } \\
\text { référence) }\end{array}$ & - & - & - & - & - & - & - & - & - & - & - & - & - & - & - & - & - & - & - & - \\
\hline $\begin{array}{l}\text { Plantations } \\
\text { d'âge } \\
\text { moyen }\end{array}$ & $-0,004$ & 0,05 & $-0,081$ & $0,935 \mathrm{~ns}$ & 3,59 & 2,83 & 1,27 & 0,20 ns & 3,24 & 0,29 & 10,97 & $0,000 * * *$ & 2,75 & 0,16 & 16,94 & $0,000 * * \star$ & 2,28 & 0,15 & 14,88 & $0,000^{* * *}$ \\
\hline $\begin{array}{l}\text { Plantations } \\
\text { âgées }\end{array}$ & $-0,551$ & 0,066 & $-8,365$ & $0,000 * * \star$ & 767 & 4,51 & 1,7 & $0,08 \mathrm{~ns}$ & 5,73 & 0,34 & 16,81 & $0,000^{\star * *}$ & 5,01 & 0,22 & 23,18 & $0,000 * * \star$ & 3,92 & 0,2 & 19,27 & $0,000^{\star * *}$ \\
\hline \multicolumn{21}{|c|}{ Effet du type de sol } \\
\hline $\begin{array}{l}\text { Vertisol } \\
\text { (catégorie de } \\
\text { référence) }\end{array}$ & - & - & - & - & - & - & - & - & - & - & - & - & - & - & - & - & - & - & - & - \\
\hline $\begin{array}{l}\text { Sol } \\
\text { ferrallitique }\end{array}$ & 0,812 & 0,042 & 19,26 & $0,000 * \star \star$ & $-6,43$ & 2,61 & $-2,46$ & $0,01^{*}$ & $-0,88$ & 0,27 & $-3,18$ & $0,001^{\star \star}$ & $-1,54$ & 0,14 & $-11,2$ & $0,000^{* * *}$ & 0,3 & 0,13 & 2,3 & $0,02^{*}$ \\
\hline $\begin{array}{l}\text { Sol } \\
\text { ferrugineux }\end{array}$ & 0,662 & 0,052 & 12,81 & $0,000^{\star \star \star}$ & $-9,4$ & 3,39 & $-2,77$ & $0,005^{\star \star}$ & 1,28 & 0,34 & 3,76 & $0,000 * \star \star$ & 0,6 & 0,17 & 3,57 & $0,000 * * \star$ & 0,12 & 0,16 & 0,76 & $0,45 \mathrm{~ns}$ \\
\hline $\begin{array}{l}\text { Sol } \\
\text { sableux }\end{array}$ & $-0,245$ & 0,085 & $-2,882$ & $0,003^{\star \star}$ & 0,87 & 5,53 & 0,16 & $0,87 \mathrm{~ns}$ & $-3,49$ & 0,44 & $-7,94$ & $0,000 * \star \star$ & $-2,23$ & 0,3 & $-7,8$ & $0,000 * \star \star$ & $-1,72$ & 0,26 & $-6,55$ & $0,000 * \star \star$ \\
\hline \multicolumn{21}{|c|}{ Interaction type de plantation et sol } \\
\hline $\begin{array}{l}\text { Plantation } \\
\text { d'âge } \\
\text { moyen } \\
\text { x sol } \\
\text { ferrugineux }\end{array}$ & $-0,192$ & 0,079 & $-2,444$ & $0,014^{*}$ & $-3,35$ & 4,87 & $-0,68$ & $0,49 \mathrm{~ns}$ & $-1,39$ & 0,45 & $-3,09$ & $0,001^{\star \star}$ & $-0,9$ & 0,26 & $-3,49$ & $0,000 * \star \star$ & $-0,66$ & 0,24 & $-2,71$ & $0,006^{\star \star}$ \\
\hline
\end{tabular}

Les éclaircies permettent d'avoir davantage d'individus de gros diamètre de $A$. auriculiformis. Les plantations jeunes, d'un âge compris entre quatre et six ans, présentent une structure diamétrique analogue, ce qui suggère qu'il est possible de prévoir une éclaircie entre quatre et six ans pour augmenter la vitesse de croissance en diamètre des arbres. Cette pratique d'éclaircie devrait éventuellement être suivie par l'élagage des arbres pour limiter les risques d'obtenir des arbres bas-branchus. Cependant, il faudra garantir une amélioration des techniques de production pour augmenter la productivité des plantations afin de répondre à une demande grandissante. Des techniques d'amélioration génétique et d'hybridation avec Acacia mangium, expérimentées dans d'autres régions avec des productivités intéressantes, pourraient être envisagées (Hung et al., 2016).

Dans les plantations âgées de 15 à 29 ans, la classe de diamètre la plus représentée est celle de 25 à $30 \mathrm{~cm}$, avec des arbres atteignant toutefois un diamètre de $50 \mathrm{~cm}$. Ces plantations sont exploitables pour des utilisations en bois d'œuvre. Les premiers produits d'éclaircie peuvent servir de bois éner- gie, et les arbres issus de futaie, de bois d'œuvre. Toutefois, il est nécessaire de compléter ces données avec les caractéristiques physico-mécaniques de $A$. auriculiformis, également déterminantes pour les différents usages du bois.

Une rotation de 15 ans pour la production de bois d'œuvre est envisageable sur sols ferrugineux, avec en moyenne deux éclaircies. L'expérimentation sur les autres types de sols permettra de déduire l'âge d'exploitabilité de A. auriculiformis pour le bois d'œuvre, sur l'ensemble des sols où cette essence est plantée au Bénin. La densité élevée des arbres d'un diamètre de 20 à $25 \mathrm{~cm}$ au sein des plantations âgées de 29 ans, couplée au faible taux d'accroissement moyen en diamètre, suggère de pratiquer plusieurs éclaircies dans les plantations avant l'âge adulte.

La distribution de Weibull montre que même les plantations âgées présentent une dominance d'individus de faible diamètre. Cela sollicite l'aménagement des plantations âgées, movennant le suivi de la croissance des arbres et la pratique régulière d'éclaircies.

Le diamètre moyen des arbres, l'accroissement moyen 
annuel, de même que les hauteurs dominante et totale, sont plus élevés sur sols ferrugineux que sur vertisols. Si l'on se réfère aux travaux de Gnanguenon-Guesse et al. (2017), relatifs à la croissance de $T$. grandis, qui représente l'essence à croissance rapide la plus utilisée comme bois d'œuvre au Bénin, on observe que $A$. auriculiformis présente un diamètre (d) moyen similaire sur vertisols. En revanche, sur sols ferrugineux, de faibles diamètres sont relevés chez $T$. grandis $(\mathrm{d}=10,5 \mathrm{~cm})$ (Gnanguenon-Guesse et al., 2017). A. auriculiformis présenterait donc une croissance supérieure au teck sur sols ferrugineux dans la zone guinéenne du Sud-Bénin.

La forte corrélation observée entre les deux méthodes de détermination du pourcentage de duramen (rondelles et carottes de bois) suggère qu'il est possible de déterminer le pourcentage de duramen à partir des carottes de bois de l'arbre, sans nécessité d'abattre les arbres échantillonnés. Le duramen est présent à quatre ans sur $A$. auriculiformis, ce qui suppose une utilisation potentielle des arbres jeunes comme bois d'œuvre.

Le pourcentage d'aubier est plus élevé, dans les plantations jeunes, sur sols ferrugineux. Selon Polge (1964), le taux d'aubier est un indicateur de fertilité de la station et de la vigueur de végétation. Un fort taux d'aubier est souvent lié à une grande surface foliaire (Rabhi et al., 2014). Nos observations indiquent que les plantations jeunes de $A$. auriculiformis sur sols ferrugineux et ferrallitiques ont une couronne plus volumineuse que les plantations sur les autres types de sols étudiés. Cette forte couronne pourrait expliquer l'accroissement annuel élevé en diamètre pour ces types de sols.

Les défauts les plus importants sur les barreaux débités sont obtenus sur les arbres de 29 ans n'ayant pas subi d'éclaircie. L'âge apparaîtrait donc comme un facteur d'augmentation de défauts dans le bois de $A$. auriculiformis. Toutefois, les coupes frauduleuses sélectives pourraient aussi bien en être la cause. Dans l'hypothèse que les arbres de meilleure qualité sont coupés frauduleusement, une réduction de la durée de rotation limiterait ces coupes abusives, qui augmentent avec l'âge des plantations.

L'âge optimal pour la séquestration de carbone de A. auriculiformis est de 20 ans au Bénin. Pour l'ensemble des types de sols au Sud-Bénin, il est possible de prévoir une exploitation de bois d'œuvre à 20 ans environ. Cette exploitation peut être anticipée sur sols ferrugineux, à Pahou, en raison de la croissance rapide des arbres sur ce type de sol. Toutefois, le suivi sur une durée plus longue des plantations établies sur les quatre types de sols permettra de mieux apprécier la situation.

\section{Conclusion}

La productivité en bois de Acacia auriculiformis varie avec le type de sol et l'âge des parcelles. Parmi les facteurs de production étudiés, le type de sol est le plus déterminant. Les sols ferrugineux présentent un indice de fertilité élevé et une productivité élevée, qui sont moindres pour les sols ferrallitiques. Il est envisageable de conduire des plantations à A. auriculiformis pour la production de bois d'œuvre, en raison des diamètres élevés obtenus à 15 ans, de la quantité de carbone stockée, et du fort pourcentage de duramen. Dans la zone guinéennene, $A$. auriculiformis présente une croissance supérieure à celle de Tectona grandis, essence à croissance rapide la plus fréquemment plantée. II importe toutefois de déterminer les propriétés anatomiques, physiques et mécaniques des bois de $A$. auriculiformis produits sur les différents types de sols, afin de statuer sur leurs potentialités d'utilisation en bois d'œuvre.

\section{Remerciements}

La Fondation internationale pour la science (FIS ; Grant I-1-D-6154-1) a subventionné en partie cette recherche par l'octroi d'une bourse attribuée au premier auteur.

\section{Références bibliographiques}

Adomou A. C., 2005. Vegetation patterns and environmental gradients in Benin: implications for biogeography and conservation. PhD thesis, Wageningen University, Wageningen, Netherlands, 150 p.

Ahoba A., Issali A. E., Gnaoré L. C., Gbogbo A. G., Kouadio M. K., 2016. Le bois du fraké issu de plantation exprime aussi de bonnes qualités technologiques. Journal of Applied Biosciences, 106 : 10215-10223. https://doi.org/10.4314/jab.v106i1.1

Charru M., Seynave I., Morneau F., Bontemps J.-D., 2010. Recent changes in forest productivity: An analysis of national forest inventory data for common beech (Fagus sylvatica L.) in north-eastern France. Forest Ecology and Management, 260: 864-874. https://doi. org/10.1016/j.foreco.2010.06.005

Dah-Dovonon V.-M., Yevide A. S. I., Hounmenou C. G., Ganglo J. C., 2013. Dynamique des plantations privées de teck (Tectona grandis L.f.) dans le département de l'Atlantique (sud Bénin) : cas de la Commune de Tori-Bossito. e-Journal of Science \& Technology, 8 (4) : 31-38. https://doi.org/10.4314/ijbcs.v5i3.72265

Dobbertin M., 2005. Tree growth as indicator of tree vitality and of tree reaction to environmental stress. European Journal of Forest Research, 124: 319-333. https://doi.org/10.1007/s10342-005-0085-3

Duan H., Cao S., Zheng H., Hu D., Lin J., Lin H., et al., 2016. Variation in the Growth Traits and Wood Properties of Chinese Fir from Six Provinces of Southern China. Forêts, 7: 192. https://doi.org/10.3390/f7090192

FAO, 2002. Quatrième réunion du sous-comité ouest et centre africain de corrélation des sols pour la mise en valeur des terres. Abomey, Bénin, 9-13 octobre 2000. http://www.fao.org/docrep/005/Y3948F/ y3948f00.htm\#toc

Fonseca W., Alice F. E., Rey-Benayas J. M., 2011. Carbon accumulation in aboveground and belowground biomass and soil of different age native forest plantations in the humid tropical lowlands of Costa Rica. New Forests, 43: 197-211. https://doi.org/10.1007/s11056-011-9273-9

Fonton N. H., Glèlè Kakai R., Rondeux J., 2002a. Étude dendrométrique d'Acacia auriculiformis A. Cunn. ex Benth. en mélange sur vertisol au Bénin. Biotechnologie, Agronomie, Société et Environnement, 6 (1) : 29-37.

Fonton H. N., Claustriaux J. J., Agbahungba G., 2002b. Performance de Acacia auriculiformis (Cunn. A.) dans le système agroforestier au Sud-Bénin. Annales des Sciences Agronomiques, 3 (1) : 65-79. https:// doi.org/10.4314/asab.v3i1.43388

Gérard J., 1991. Gutta Percha, ironwood et lancewood, trois nouveaux bois précieux nord-australiens, un exemple de sélection technologique et de promotion d'essences secondaires tropicales. Bois et Forêts des tropiques, $228: 63-73$. https://doi.org/10.19182/bft2014.320. a20548

Gnahoua G. M., Louppe D., 2003. Acacia auriculiformis. Cirad, 2 p.

Gnanguenon-Guesse D., Nounagnon G. S., Aoudji A. K. N., Ganglo J. C., 2017. Étude des caractéristiques structurales des teckeraies en fonction de l'âge et du type de sol au Sud et au Centre Bénin. International Journal of Biological and Chemical Sciences, 11 (5) : 2119-2132. https://doi.org/10.4314/iibcs.v11i5.15

Griffin A. R., Midgley S. J., Bush D., Cunningham P. J., Rinaudo A. T., 2011. Global uses of Australian acacias - recent trends and future prospects. Diversity and Distributions, 17: 837-847. https://doi. org/10.1111/i.1472-4642.2011.00814.X 
Hai P. H., Jansson G., Harwood C., Hannrup B., Thinh H. H., 2008. Genetic variation in growth, stem straightness and branch thickness in clonal trials of Acacia auriculiformis at three contrasting sites in Vietnam. Forest Ecology and Management, 255 (1): 156-167. https://doi. org/10.1016/j.foreco.2007.09.017

Hai P., Hannrup B., Harwood C., Jansson G., Ban D. V., 2010. Wood stiffness and strength as selection traits for sawn timber in Acacia auriculiformis. Canadian Journal of Forest Research, 40 (2): 322-329. https:// doi.org/10.1139/x09-191

Hung T. T., Almeida A. C., Eyles A., Mohammed C., 2016. Predicting productivity of Acacia hybrid plantations for a range of climates and soils in Vietnam. Forest Ecology and Management, 367: 97-111. https://doi. org/10.1016/i.foreco.2016.02.030

Husch B., Beers T. W., Kershaw J. A. K. R., 2002. Forest Mensuration. 4th edition. Wiley, $456 \mathrm{p}$.

Jha K. K., 2018. Root carbon sequestration and its efficacy in forestry and agroforestry systems: A case of Populus euramericana l-214 cultivated in Mediterranean condition. Notulae Scientia Biologicae, 10 (1): 68-78. https://doi.org/10.15835/nsb10110181

Kataki R., Konwer D., 2002. Fuelwood characteristics of indigenous tree species of north-east India. Biomass and Bioenergy, 22 (6): 433-437. https://doi.org/10.1016/s0961-9534(02)00026-0

Kokutse A. D., Baillères H., Stokes A., Kokou K., 2004. Proportion and quality of heartwood in Togolese teak (Tectona grandis L.f). Forest Ecology and Management, 189: 37-48. https://doi.org/10.1016/i. foreco.2003.07.041

Maslin B. R., 2014. Two new species of Acacia (Fabaceae: Mimosoideae) with conservation significance from Banded Iron Formation ranges in the vicinity of Koolyanobbing, Western Australia. Nuytsia, 24: 131-138. https://florabase.dpaw.wa.gov.au/nuytsia/article/719

Nemani R. R, Keeling C. D., Hashimoto H., Jolly W. M., Piper S. C., Tucker C. J., et al., 2003. Climate-driven increases in global terrestrial net primary production from 1982 to 1999. Science, 300: 1560-1563. https://doi.org/10.1126/science.1082750

N’Guessan K. A., Voui Bi Bianuvrin N. B., Traoré S., Tapé-Bi F. A., 2015. Productivité maximale et âge d'exploitabilité technique de Tectona grandis (teck) en fonction de la fertilité des stations en Côte d'ivoire. Journal of Applied Biosciences, 95: 9003-9014. https://doi. org/10.4314/jab.v95i1.10

N'Guessan A. K., Assande A., Issali A. E., Vouibi N. B., Yeo O., 2016. Comment régénérer naturellement une forêt en Côte d'Ivoire ? Journal of Applied Biosciences, 105 : 10085-10091. https://doi.org/10.4314/ jab.v105i1.4

Ostadhashemi R., Shahraji R. T., Roehle H., Limaei M. S., 2014. Estimation of biomass and carbon storage of tree plantations in northern Iran. Journal of Forest Science, 60 (9): 363-371. https://doi. org/10.17221/55/2014-jfs

Owona Ndongo P.-A., Peltier R., Linjouom I., Louppe D., Smektala G., Beligné V., et al., 2009. Plantations de bois d'œuvre en zone équatoriale africaine : cas de l'arboretum de l'Enef de Mbalmayo au sud du Cameroun. Bois et Forêts des Tropiques, 299 (1) : 37-48. https://doi. org/10.19182/bft2009.299.a20421

Peltier R., Forkong C. N., Ntoupka M., Manlay R., Henry M., Morillon V., 2007. Évaluation du stock de carbone et de la productivité en bois d'un parc à karités du Nord-Cameroun. Bois et Forêts des Tropiques, 294 (4) : 39 -50. https://doi.org/10.19182/bft2012.311.a20507

Polge H., 1964. Délimitation de l'aubier et du bois de cœur par analyse densitométrique de clichés radiographiques. Annales des Sciences Forestières, 21 (4) : 605-623. https://doi.org/10.1051/ forest/19640404

Rabhi K., Tafer M., Messaoudene M., 2014. Évolution des proportions d'aubier et de duramen du cèdre de l'Atlas, Cedrus atlantica Manetti, en Algérie. Bois et Forêts des Tropiques, 322 (4) : 77-87. https://doi. org/10.19182/bft2014.322.a31238

Rondeux J., 1999. La mesure des arbres et des peuplements forestiers. Presses Agronomiques de Gembloux, Belgique, 521 p.
Seynave I., Gégout J.-C., Hervé J.-C., Dhôte J.-F., 2008. Is the spatial distribution of European beech (Fagus sylvatica L.) limited by its potential height growth? Journal of Biogeography, 35: 1851-1862. https://doi. org/10.1111/j.1365-2699.2008.01930.x

Sokpon N., Biaou S. H., Ouinsavi C., Hunhyet O., 2006. Bases techniques pour une gestion durable des forêts claires du Nord-Bénin : rotation, diamètre minimal d'exploitabilité et régénération. Bois et Forêts des Tropiques, $287:$ 45-57. https://doi.org/10.19182/bft2012.312. a20501

Sokpon N., Ouinsavi C., 2004. Gestion des plantations de Khaya senegalensis au Bénin. Bois et Forêts des Tropiques, 279 (1) : 37-46. https://doi.org/10.19182/bft2004.279.a20247

Tandjiekpon A. M., Dah-Dovonon J. Z., 1997. Régénération naturelle par rejet de souche de Acacia auriculiformis A. Cunn. ex Benth. Bulletin de la Recherche Agronomique, 20 : 18-31. http://www.slire.net/download/1112/tandjiekpon bra 020 1997-2.pdf

Volkoff B., Willaime P., 1976. Carte pédologique de reconnaissance de la République Populaire du Bénin à 1/200 000 : Feuille de Porto Novo. Paris, France, Orstom, Notice explicative 66 (1), 39 p. http:// horizon.documentation.ird.fr/exl-doc/pleins textes/pleins textes_5/ notexp/08431.pdf

\section{Tonouéwa et al. - Contribution des auteurs RÔLE DU CONTRIBUTEUR NOMS DES AUTEURS}

\begin{tabular}{|c|c|}
\hline Conceptualisation & J. F. M. F. Tonouéwa \\
\hline Gestion des données & J. F. M. F. Tonouéwa \\
\hline Analyse formelle & $\begin{array}{l}\text { S.S. H. Biaou, } \\
\text { J. F. M. F. Tonouéwa }\end{array}$ \\
\hline $\begin{array}{l}\text { Acquisition du } \\
\text { financement }\end{array}$ & J. F. M. F. Tonouéwa \\
\hline Enquête et investigation & J. F. M. F. Tonouéwa \\
\hline Méthodologie & $\begin{array}{l}\text { S. S. H. Biaou, } \\
\text { J. F. M. F. Tonouéwa }\end{array}$ \\
\hline Gestion de projet & $\begin{array}{l}\text { S.S. H. Biaou, } \\
\text { E.P. S. Assédé }\end{array}$ \\
\hline Ressources & $\begin{array}{l}\text { S. S. H. Biaou, } \\
\text { E. P. S. Assédé, A. K. Natta }\end{array}$ \\
\hline Logiciels & S.S. H. Biaou \\
\hline Supervision & $\begin{array}{l}\text { S. S. H. Biaou, } \\
\text { E. P. S. Assédé, A. K. Natta }\end{array}$ \\
\hline Validation & $\begin{array}{l}\text { S. S. H. Biaou, } \\
\text { E. P. S. Assédé, A. K. Natta }\end{array}$ \\
\hline Visualisation & $\begin{array}{l}\text { J. F. M. F. Tonouéwa, } \\
\text { S. S. H. Biaou, } \\
\text { E. P. S. Assédé, A. K. Natta }\end{array}$ \\
\hline $\begin{array}{l}\text { Écriture - Préparation } \\
\text { de l'ébauche originale }\end{array}$ & $\begin{array}{l}\text { J. F. M. F. Tonouéwa, } \\
\text { S. S. H. Biaou, } \\
\text { E. P. S. Assédé, A. K. Natta }\end{array}$ \\
\hline $\begin{array}{l}\text { Écriture - Révision } \\
\text { et édition }\end{array}$ & $\begin{array}{l}\text { J. F. M. F. Tonouéwa, } \\
\text { S. S. H. Biaou, } \\
\text { E. P. S. Assédé, A. K. Natta }\end{array}$ \\
\hline
\end{tabular}

Bois et Forêts des Tropiques - Revue scientifique du Cirad
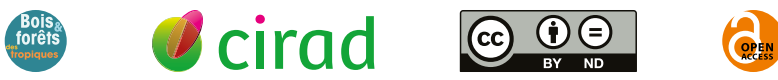

Cirad - Campus international de Baillarguet, 34398 Montpellier Cedex 5, France - Contact : bft@cirad.fr - ISSN : L-0006-579X 